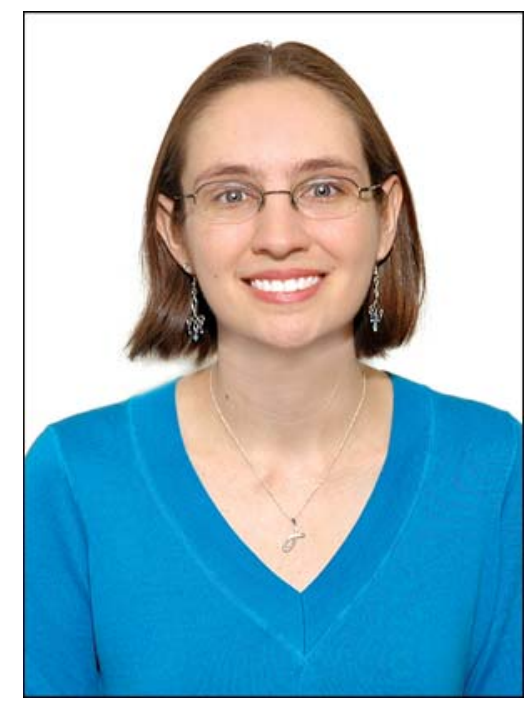

Johanna L. Gribble, MA

\section{From the Editor's Desk}

\section{Thank you to JRRD reviewers}

Another successful year has passed for the Journal of Rehabilitation Research and Development (JRRD), and we are looking forward to 2013. We could not have accomplished this without the support of our authors, readers, and reviewers. We would especially like to thank the members of our Editorial Board and the 521 reviewers who dedicated their time, knowledge, and energy toward reviewing each submission and ensuring the continued quality of our content.

This year, JRRD will be celebrating its 50th anniversary with the Then \& Now project. In this issue, we will revisit an editorial from 1965 and Dr. Lucille Beck, Chief Consultant, Office of Rehabilitation Services, Office of Patient Care Services, Veterans Health Administration, will comment on 65 years of progress at the VA Prosthetics and Sensory Aids Service. Each issue of volume 50 will also feature highlights from early articles of the Bulletin of Prosthetics Research with commentary by either past or current specialists in that field.

Over the past 2 years, JRRD has published single-topic issues on the road ahead for rehabilitation robotics, upper-limb prosthetics, posttraumatic stress disorder clinical guidelines, and sensory and communication disorders in traumatic brain injury. We have also featured single-topic sections on wound care practice, wheelchair engineering, and outcome measures in rehabilitation. This year, look for a single-topic issue on outcome measures for veterans with lower-limb loss.

As part of our "Going Green” initiative and to comply with Executive Order 13589 (Promoting Efficient Spending), we are endeavoring to limit hardcopy production of JRRD in order to reduce paper use by 20 percent. $J R R D$ will continue to be freely accessible via our Web site (www.rehab.research.va.gov/jrrd). Beginning July 1, 2013, a new hardcopy subscription of the journal can be purchased from the U.S. Government Bookstore (bookstore.gpo.gov, search “JRRD”). In coordination with GPO, JRRD will also be available for purchase for easy download to your tablet or smartphone from Zinio, where articles can be viewed in either PDF or free-flow text format (www.zinio.com).

If you are an author or reviewer, please take a moment to review our updated Submission Guidelines and Editorial Policies. Specifically, we have updated sections involving self-plagiarism: what it is and how to avoid it. For more detailed information on the topics of plagiarism and self-plagiarism, see the "From the Editor's Desk" in volume 49, issue 8 of JRRD [1].

As part of our effort to reach and inform more veterans, researchers, and clinicians worldwide, we have expanded our foreign language JRRD At a Glance offerings (available on our Web site). Translations of every layperson summary are available in Spanish, traditional Chinese, and simplified Chinese. In the summer of 2012, JRRD was part of an international book tour to 
China as part of The Charlesworth Group’s Academic Journal Exhibition 2012. The tour covered approximately 70 percent of the Chinese print subscription market. Issues of the journal were displayed in Wuhan, Shanghai, and Shenzhen and at the Beijing International Book Fair-Overseas Periodical Show. The proactive JRRD outreach into this arena is a direct result of recent publications in Science and Nature that report on the rising Asian influence in scientific research and resultant publications [2-3]. Participating in this journal exhibition has substantially increased both our readership and the number of submissions from the Pacific Rim countries.

Our social media offerings online continue to grow. JRRD is available on both Facebook (http://jrrdjournal.facebook.com) and Twitter (@jrrdeditor), and both are updated regularly with rehabilitation news. We encourage everyone to "like" or "follow" us to keep up to date with JRRD. This year, the staff also developed and animated a short video called JRRD 180, which provides an excellent introduction to the journal and its role in the rehabilitation research community. Finally, podcasts with information about all the articles in each issue are available for free in the iTunes store and on the JRRD Web site. Episodes are available in both English and Spanish.

The estimated number of downloads from the JRRD Web site in 2002 was approximately 500,000. In 2012, we had 9.6 million! The journal has come far in 10 years, and we can't wait to see how far we will grow in the next decade.

\section{Johanna L. Gribble, MA}

Technical Writer-Editor, JRRD

http://dx.doi.org/10.1682/JRRD.2013.02.0047

1. Horrom TA. The perils of copy and paste: Plagiarism in scientific publishing. J Rehabil Res Dev. 2012;49(8):vii-xii. http://dx.doi.org/10.1682/JRRD.2012.09.0165

2. Reich ES. Research in Asia heats up. Nature. 2012;481(7382):420. [PMID:22281571] http://dx.doi.org/10.1038/481420a

3. Mervis J. Science Indicators 2012. Report notes China’s influence in emerging Asian science zone. Science. 2012;335(6066):274-75. [PMID:22267783] http://dx.doi.org/10.1126/science.335.6066.274

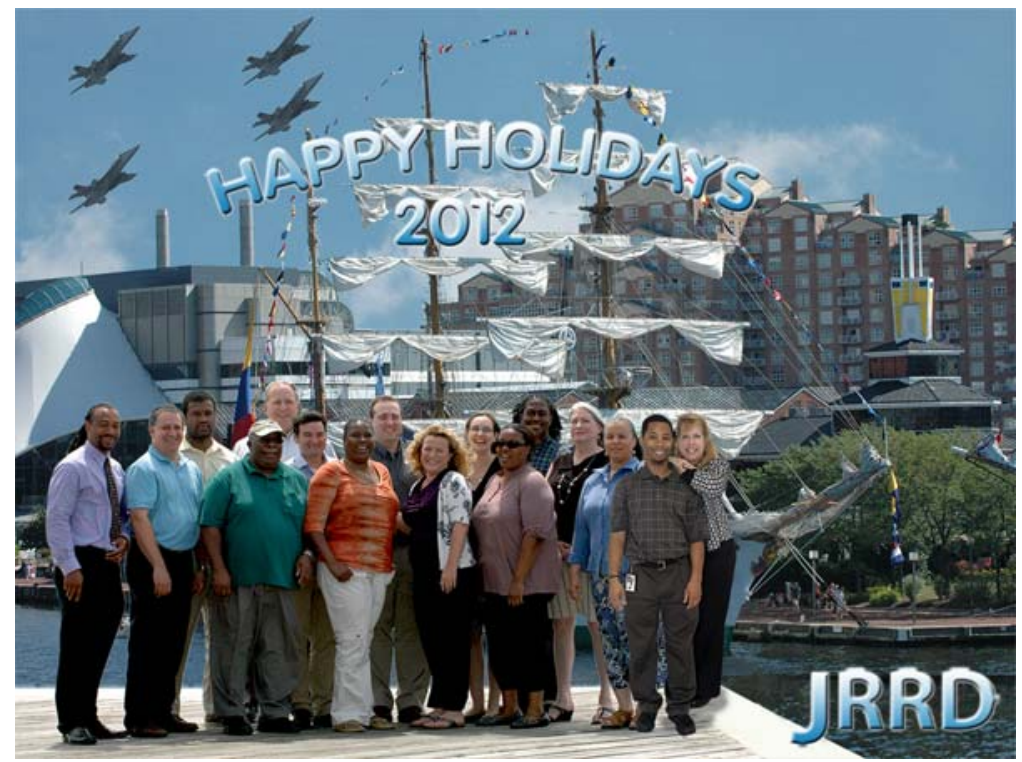

From Left to Right: Robert Williams, Ken Frager, Milton Gholton, Author Parker, Lloyd Tinker, Dave Bartlinski, Celeste Anderson, Tristan Horram, Maryn Rosenberg, Johanna Gribble, Carol Roberts, Justin James, Margurite Shipley, Lisa, Finklea, Rodney Baylor, and Stacie Yuhasz (missing from photo Rebecca Torres). 


\section{JRRD 2012 Peer Reviewers}

A

Abrams, Gary

Abrams, Harvey

Acharya, Aninda

Ajiboye, Bolu

Akbari, Asghar

Akin, Faith

Akins, Jonathan

Akpolat, V.

Alaqtash, Murah

Alghadiri, Ahmad

Alguren, Beatrix

Al-Khawaja, Imad

Alsanak, Serap

Amatachaya, Samata

Amiri, Pouya

Amiri-Khorasani,

Mohammadtaghi

Ammer, William

Ananda Pandian, Ponsekar

Annicchiarico, Roberta

Anson, Denis

Apatsidis, Dimitrios

Archambault, Philippe

Armstrong, Michael

Aruin, Alexander

Asahina, Masato

Ashford, John

Atkins, Diane

Audu, Musa

\section{B}

Babyar, Suzanne

Bach, Carole Ann

Bachus, Kent

Backus, Deborah

Bair, Byron

Barfield, J. P.

Barker, Felix

Barlow-Ogden, Kristen

Barnett, Scott

Baron, Mark

Bates, Barbara

Bayat, Mohammad

Beaver, Bonnie

Becker, Deborah

Beckham, Jean

Belkonen, Stacy
Bell, Edward

Bell, Morris

Bennett, Susan

Bernardy, Nancy

Bethoux, Francois

Betz, Kendra

Bever, Christopher

Bickel, C. Scott

Biering-Sorensen, Fin

Bijak, Manfred

Bilodeau, Martin

Birbaumer, Niels

Bishop, Mark

Bloebaum, Roy

Bohannon, Richard

Boissy, Patrick

Bond, Gary

Bourdette, Dennis

Boutwell, Erin

Bowden, Mark

Boyle, Mary

Bradham, Douglas

Brandt, Åse

Brenner, Lisa

Brienza, David

Brown, Karl

Brown, Marybeth

Brüggemann, Gert-Peter

Bufka, Lynn

Burdea, Grigore

Burger, Helena

Burkard, Robert

Bushnik, Tamara

Byrnes, K. R.

\section{C}

Cameron, Michelle

Campillo, Philippe

Capehart, Bruce

Carlson, Lawrence

Carre, Emilie

Carrington, Jane

Casey, Kevin

Caspers, Carl

Castillo, Diane

Caughlan, Carly

Caves, Kevin

Chae, John

Chang, Ya-Ju
Chang, Young-Hui

Chelimsky, Thomas

Chen, John

Cheng, Chih Hsiu

Chichoine, David

Childers, Lee

Chin, Takaaki

Chong W. Lee, Alan

Choppa, Tony

Chung, Duc

Cifu, David

Ciuffreda, Kenneth

Clark, Dave

Collins, Diane

Colombo, Giorgio

Constantinou, Chris

Cooper, Robin

Coote, Susan

Corbett, Elaine

Corrigan, John

Côté, Marie-Pascale

Couch Jr, James

Coughlin, Steven

Coupaud, Sylvie

Cowan, Rachel

Culpepper, William

Curtin, Catherine

Cutter, Gary

\section{D}

Daher, Pamela

Daly, Janis

Daly, Wayne

Dankmeyer, Charles

Darter, Benjamin

Davis, Brian

Davis, Christine

Day, Kristin

De Bruin, Eling Douwe

De Groot, Sonja

de la Cruz Torres, Blanca

Dean, Jesse

Decker, Suzanne

Dekker, Rienk

del Ama, Antonio

Demir, Hüseyin

Demirtas, Raziye

Dennis, Kyle

Deshpande, Shripad B.
Desroches, Guillaume

Detrembleur, Christine

DeViva, Jason

Diaz-Arrastia, Ramon

Dicianno, Brad

Dickin, Clark

DiGiovine, Carmen

Dijkers, Marcel

Diserens, Karin

Donaldson, Nick

Downes, Barry

Drebing, Charles

Dtkach, Dennis

Duraski, Sylvia

Durfee, William

E

Eapen, Blessen

Edmonds, Lisa

Edwards, Mark

Ehde, Dawn

Ehrlich-Jones, Linda

Ekberg, Olle

Ellis, Charles

Elnitsky, Christine

Eom, Gwang-Moon

Erbes, Christopher

Erdemir, Ahmet

Eshraghi, Arezoo

\section{F}

Falvo, Michael

Fatone, Stefania

Faul, Mark

Feng, Wayne

Fergason, John

Ferris, Daniel

Figoni, Stephen

Filipi, Mary

Foto, James

Franceschini, Marco

Frank, Andrew

Freitas, Paulo

French, Dustin

French, Louis

Friedman, Matthew

Friel, Karen

Frisina, Robert 
Frossard, Laurent

Frost, Karen

Fuglevand, Andrew

Fujioka, Y.

Fulk, George

G

Gailey, Robert

Gambel, Jeffrey

Gard, Steven

Garvin, Jennifer

Gater, David

Gefen, Amit

Gerschutz, Maria

Gholizadeh, Hossein

Ghotbi, Nastaran

Gibson, Carri-Ann

Gifford, Rene

Gil-Agudo, Angel

Gillette, Jason

Glynn, Shirley

Goetz, Lance

Gojanovic, Boris

Goldberg, Gary

Goldfarb, Michael

Gonzalez-Rothi, Leslie

Gonzenbach, Stephen

Goodnough, Jason

Goodrich, Gregory

Goodson, Jason

Gorce, Philippe

Greenwald, Richard

Griffin, Murray

Griffith, James

Groer, Shirley

Gulliver, Suzy

\section{H}

Hafner, Brian

Hagberg, Kerstin

Hahn, Michael

Hall, Carmen

Hamblin, Jessica

Hannold, Lisa

Hansen, Andrew

Harkness, Laurie

Harlaar, Jaap

Haselkorn, Jodie

Haubert, Lisa

Haun, Andrew
Hayner, Stephanie

Hebert, Jacqueline

Heckathorne, Craig

Henriksen, Arne

Henzel, M.

Hesse, Stefan

Heyn, Patricia

Hicks, Audrey

Highsmith, M. Jason

Hill, Wendy

Ho, Chester

Hochberg, Leigh

Hof, At

Hoffer, Michael

Holmberg, Janene

Hooper, Rebecca

Houdijk, Han

Howell, Jared

Howell, Paul

Hsieh, Ru-Lan

Huang, Helen

Hubbard, Shiela

Hubbard-Winkler, Sandra

Hughes, Chris

Hunt, Marcia

\section{I}

Ishikawa, Saori

Iverson, Katherine

J

Jacelon, Cynthia

Jackson, James

Jacobs, Reinhilde

Jan, Mehrholz

Jansen, Mary

Janssen, Thomas

Jayaraman, Arun

Johannesson, Anton

John, Emmanuel

Johnson, Gregory

Johnson, Shawn

Johnston, Therese

Joines, Sharon

Jutai, Jeff

K

Kaelin, Darryl

Kahle, Jason T.

Kairy, Dahlia
Kalra, Lalit

Kapp, Susan

Karimi, Hossein

Karmarkar, Amol

Kaufman, Kenton

Kautz, Steven

Kerckhofs, Eric

Kesselring, Jürg

Kirby, Ronald

Kivlahan, Daniel

Kloth, Luther

Klute, Glenn K.

Knutson, Jayme

Kobayashi, Toshiki

Koester, Heidi

Kohler, Friedbert

Koontz, Alicia

Krassioukov, Andrei

Kraus, Nina

Krol, Lukasz

Krouskop, Thomas

Kuo, Chung-Hsien

Kyberd, Peter

\section{L}

Lake, Chris

Lam, S. C.

Lambercy, Olivier

Lamiani, Giulia

Lamoth, C. J.

Landel, Robert

Lang, Catherine

Lareau, Suzanne

LaRocca, Nicholas

Lauer, Richard

Laws, Michael

Ledoux, William

Lee, Ken

Lee, Peter

Lee, Sang Wook

Lemaire, Edward

Leon-Carrion, Jose

Leong, Chau-Peng

Leskela, Jennie

Leung, Aaron

Levin, Oron

Levitt, Harry

Lew, Henry

Lewis, Gwyn

Lin, Vernon
Lindblom, Hanna

Ling, Geoffrey

Lo, Hsin-Chang

Logue, Melanie

Lonsbury-Martin, Brenda

Losier, Yves

Lowman, John

Lung, Chi-wen

Lutz, Barbara

\section{M}

Magder, Laurence

Mailey, Emily

Mak, Arthur

Malcolm, Matt

Manal, Kurt

Mantri, Sneha S.

Martin-Harris, Bonnie

Marx, Brian

Masani, Kei

Massie, Crystal

Massof, Robert

Maurer, Chris

Mavroidis, Constantinos

McCoy, Kathleen

McDonald, John

McGarry, Anthony

McMillan, Garnett

McNamee, Shane

Meier, Margrit

Messinger, Seth

Miller, Deborah

Miller, Laura

Mitsch, Sarah

Miyazaki, Shogo

Monaghan, Colleen

Montano, Joseph

Montesinos, Luis

Moreland, Leslie

Moreno, Juan

Morey, Miriam

Morley, James

Morris, Meg

Morrison, Steve

Motl, Robert

Mulcahey, M. J.

Munih, Marko

Murray, Kevin

Musson, Nan

Myers, Paula 


\section{$\mathbf{N}$}

Nadeau, Stephen

Najafi, Bijan

Neptune, Richard

Neumann, Edward

Ng, Alex

Nilsson, Michael

Norris, Fran

\section{O}

O’Brien, Erin

Olney, Christine

Oomens, Cees

Orcutt, James

Ortiz, Jose

Otten, Bert

\section{$\mathbf{P}$}

Packer, Mark

Padua, Luca

Pai, Ajit

Paillard, Thierry

Papazis, Janet

Pape, Theresa

Paquin, Gregory

Park, Hyung-Soon

Parko, Karen

Pasqui, Viviane

Pasquina, Paul

Patterson, Kara

Peak, Margaret

Pearlman, Jonathan

Perell-Gerson, Karen

Perr, Anita

Perrin, Paul

Peterson, Matthew

Peterson, Michelle

Petrone, Nicole

Pitkin, Mark

Plettenburg, Dick

Popovic, Dejan

Portnoy, Sigal

Post, Marcel

Postema, Klaas

Pugh, Mary

\section{$\mathbf{R}$}

Rammelt, Stefan

Ramsey, Mitchell

Ramstrand, Nerrolyn

Reisman, Darcy

Resnik, Linda

Ribas, Juan

Rizzo, Albert

Robinson, Chris

Rosenbaum Chou, Teri

Roth, Elliot

Ruff, Robert

Rusaw, David

Ruzek, Josef

S

Saban, Karen

Sanders, Joan

Sawatzky, Bonita

Sayer, Nina

Sayers, Steven

Schein, Richard

Scherbina, Konstantin

Schmid, Arlene

Schnurr, Paula

Schoeman, Marlene

Schoenfeld, Frank

Scholten, Joel

Schutt, Russell

Sears, Christine

Selçuk, C. T.

Shafizadeh, Mohsen

Shapiro, William

Shea, Kimberly

Sherwood, Arthur

Shi, Jun

Shields, Richard

Shorr, Ronald

Sidani, Souraya

Sloan, Denise

Smaldino, Joseph

Soede, Thijs

Sonenblum, Sharon

Spence, William

Sporner, Michelle
Sprigle, Stephen

Stampas, Argyrios

Stephenson, Jennifer

Stern, Stephen

Stienen, Arno

Storey, Christopher

Strayer, Jonathan

Stubblefield, Kathy

Suhr, Julie

Supan, Terry

Sweetow, Robert

Szeplaki, Catherine

Szlyk, Janet

\section{$\mathbf{T}$}

Tai, Changfeng

Taub, Edward

Taylor, Brent

Taylor, Dawn

Taylor, Paul

Thomas, Floria

Thorne, Sally

Thorp, Steven

Timberlake, George

Toombs, James

Torkamen, Giti

Townsend, John

Toyinbo, Peter

Triolo, Ronald

Tsai, Jack

Tsuga, Kazuhiro

Turner, Christopher

Turner, Duncan

Twamley, Elizabeth

Tyler, Richard

$\mathbf{U}$

Uswatte, Gitendra

V

Van Beek, Guus

Van Bennekom, Coen

Van der Woude, Lucas

van Drongelen, Stefan

Verrier, Molly
Vervloed, Mathijs

W

Wade, Charles

Walden, Therese

Walker, Lloyd

Wallin, Mitchell

Waltzman, Susan

Wang, Chung-Li

Wang, Hongwu

Wang, Rosalie

Wang, Tyng-Guey

Watson, Tim

Wayne, Jennifer

Weir, Richard

Weisman, Gerald

Wendland, Deborah

Werner, Cordula

West, Alan

White, Andrea

Wilkin, Jason

Willems, Jaap

Williams, T. Walley

Wilson, Richard

Winge, Kristian

Wolf, Erik

Woodward, Steven

Wurdeman, Shane

$\mathbf{X}$

Xydakis, Michael

$\mathbf{Y}$

Young, Heather

Yuhasz, Stacieann

Z

Zayeri, Farid

Zheng, Yong-Ping

Zissimopoulos, Angelika 
